\title{
Numerische Aspekte einer Mechanik-Aufgabe
}

\author{
Rainer Berghaus, Achim Kehrein
}

\section{Zusammenfassung}

Die vorliegende Arbeit entstand aus einem einfachen Mechanik-Problem, das auf eine trigonometrische Gleichung führte. Wir vergleichen am Beispiel dieses Problems verschiedene numerische Verfahren miteinander.

Das Hauptziel dieser Arbeit ist nicht die Lösung der Gleichung - sie kann sogar exakt ermittelt werden -, sondern eine Darstellung am konkreten Beispiel, wie man numerische Lösungen gewinnen kann. Insbesondere soll die Bedeutung von Bildern für die numerischen Verfahren betont werden.

Schließlich werden einige praktische Aspekte diskutiert, die nur selten konkret in der Literatur beschrieben werden. Beispielsweise bewirkt die schlechte Konditionierung des Problems, dass das Bisektionsverfahren keine eindeutige Lösung im Rahmen der Rechengenauigkeit besitzt.

\section{Abstract}

This paper is motivated by a mechanics problem and its trigonometric equation. Based on this problem we compare several numerical solution techniques.

The paper's purpose is not simply the solution of an equation - there is even an exact solution - but a detailed presentation of an example how to obtain a numerical solution. In particular, the presentation stresses the importance of pictures in supporting numerical methods.

Moreover the paper discusses some practical aspects that rarely appear in the literature. The bisection method, for instance, fails to produce a unique solution with respect to a given computational accuracy.

\section{Das mechanische Problem}

Gegeben sei ein System (Abb. 1) zweier gleich großer Massen $[m]$, die über ein Seil verbunden sind und einer Gewichtskraft $F_{G}$ unterliegen. Das Seil wird über eine Rolle umgelenkt, so dass eine Masse frei hängt und die zweite auf einer geneigten Ebene liegt. Gesucht wird der Grenzwinkel $\alpha_{\text {grenz }}$, bei dem die Haftreibung $F_{R}$ an der Masse auf der schiefen Ebene das System gerade noch im Gleichgewicht hält. Wir nehmen an, dass die Haftreibung proportional zur Normalkraft ist, $F_{R}=\boldsymbol{\mu}$. $F_{N}$, und für den Haftreibungskoeffizienten $0<\boldsymbol{\mu}<1$ gilt. In den meisten praktischen Fällen ist das experimentell nachweisbar (Hibbeler, 2004, Abschnitt 8.1). Das Seil und die Umlenkrolle werden hingegen als reibungsfrei und masselos angenommen.

Liegt die geneigte Ebene im Extremfall horizontal, so zieht die frei hängende Masse die aufliegende in Richtung der Rolle. Dazu benötigt man $\boldsymbol{\mu}<1$. Wird der Neigungswinkel $\alpha$ größer, so wird die Reibung kleiner und die der Bewegung entgegenwirkende Hangabtriebskraft größer. Im anderen Extremfall, bei $\alpha=90^{\circ}$, ist die Reibung $F_{R}=\boldsymbol{\mu} \cdot F_{N}=0$, weil keine Normalkraft

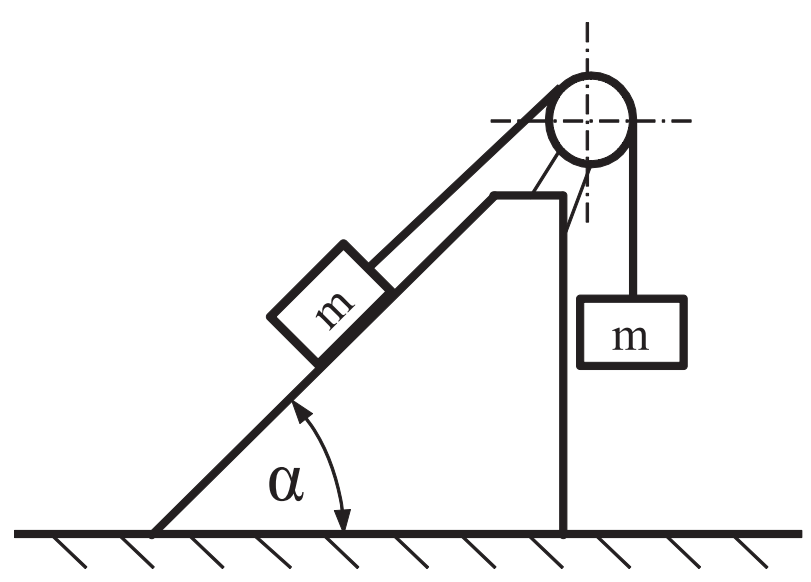

Abb. 1: mechanisches Problem

$F_{N}$ die Reibflächen gegeneinander drückt. Dann befindet sich das System im indifferenten Gleichgewicht, da auf beiden Seiten der Rolle eine gleich schwere Masse frei hängt. Da der Haftreibungskoeffizient $\boldsymbol{\mu}>$ 0 ist, gibt es einen Bereich für den Neigungswinkel der Ebene zwischen $\alpha_{\text {grenz }}$ und $90^{\circ}$, im dem die aufliegende Masse nicht von der frei hängenden in Bewegung gesetzt wird. 
1.1 Mechanische Lösung

Zunächst werden die beiden Massen und die Rolle freigeschnitten und die Gleichgewichtsbedingungen für die einzelnen Körper aufgestellt.

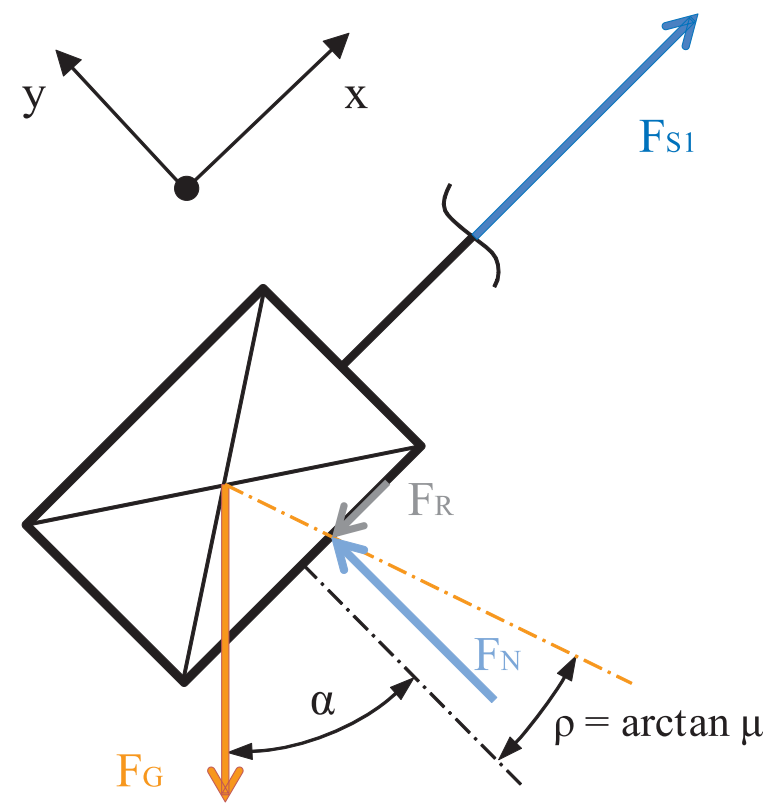

Abb. 2: aufliegende Masse

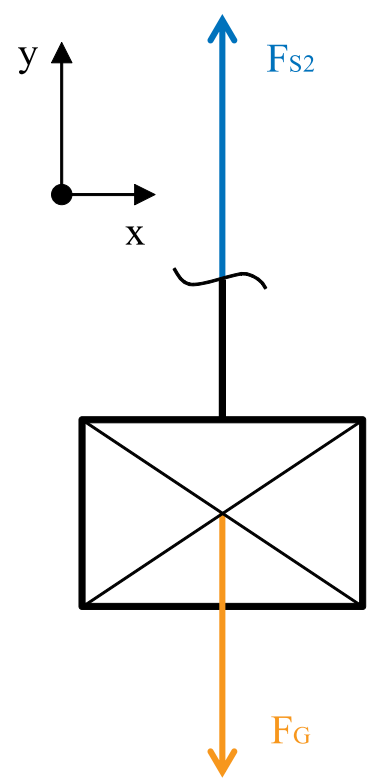

Abb. 3: frei hängende Masse

Die Summe aller an der aufliegenden Masse angreifenden Kräfte ist Null, (Das Koordinatensystem liege parallel zur Seilkraft $F_{\text {S1 }}$, s. Abb. 2)

$\overrightarrow{0}=\vec{F}_{S 1}+\vec{F}_{R}+\vec{F}_{N}+\vec{F}_{G}$

$\overrightarrow{0}=\left(\begin{array}{c}F_{S 1} \\ 0\end{array}\right)+\left(\begin{array}{c}-F_{R} \\ 0\end{array}\right)+\left(\begin{array}{c}0 \\ F_{N}\end{array}\right)+\left|\vec{F}_{G}\right|\left(\begin{array}{l}-\sin \alpha \\ -\cos \alpha\end{array}\right)$

wobei $F_{R}=\boldsymbol{\mu} \cdot F_{N}$ und $F_{G}=m \cdot g$ sind.

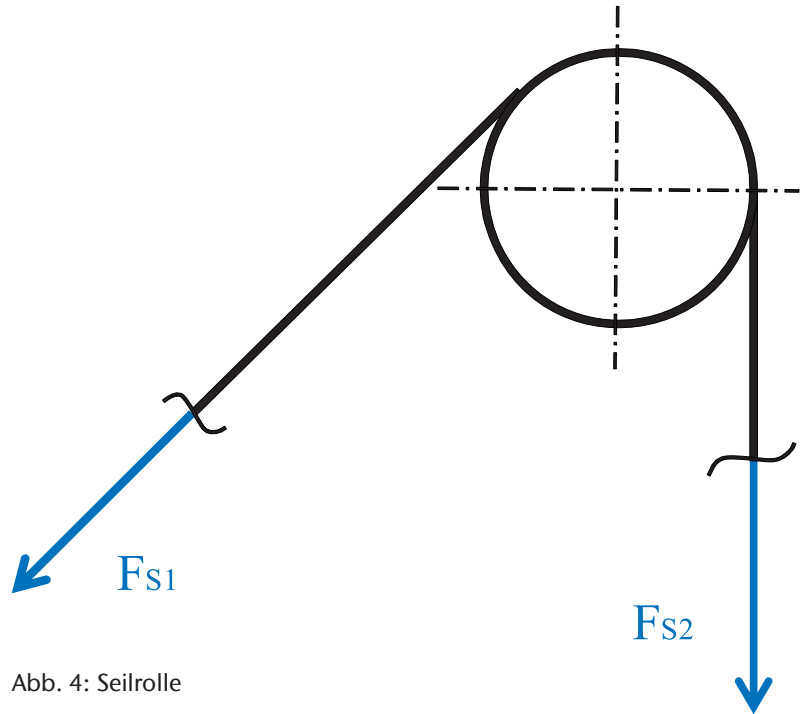

Für die Y-Komponente folgt daraus $F_{N}=F_{G} \cdot \cos \alpha$ und für die X-Komponente wird aus $F_{S 1}=F_{R}+F_{G} \sin \alpha$ durch Einsetzen der drei vorhergehenden Formeln:

$F_{S 1}=m \cdot g \cdot(\mu \cdot \cos \alpha+\sin \alpha)$

Auch an der frei hängenden Masse muss die Summe aller Kräfte Null sein. (Das Koordinatensystem liege parallel zur Seilkraft $F_{\mathrm{S} 2}$, S. Abb. 3)

$\overrightarrow{0}=\vec{F}_{S 2}+\vec{F}_{G}=\left(\begin{array}{c}0 \\ F_{S 2}\end{array}\right)+\left(\begin{array}{c}0 \\ -F_{G}\end{array}\right)$

woraus mit $F_{G}=m \cdot g$ folgt, dass $F_{S 2}=m \cdot g$.

Das Momentengleichgewicht um den Mittelpunkt der Rolle ergibt: $\overrightarrow{0}=\vec{r}_{1} \times \vec{F}_{S 1}+\vec{r}_{2} \times \vec{F}_{S 2}$

Da die Seilkräfte tangential an der Rolle liegen, sind sie senkrecht zu den Radien. Die Hebelarme der Seilkräfte haben somit beide den Betrag r. Die sich ergebenden Momente sind entgegengesetzt und liefern $F_{S 1}=F_{S 2}$. Das entspricht der einfachen Überlegung: Wenn die Rolle reibungsfrei ist, sind die Seilkräfte gleich (vgl. Abb. 4).

Setzt man nun in $F_{S 1}=F_{S 2}$ die Seilkräfte ein, erhält man eine Gleichung, die nur vom Grenzwinkel $\alpha_{\text {grenz }}$ und der Senkrechten $\left(\alpha=90^{\circ}\right)$ erfüllt wird.

$m \cdot g \cdot(\mu \cdot \cos \alpha+\sin \alpha)=m \cdot g$

Das mechanische Problem führt also auf die Gleichung $\mu \cdot \cos \alpha+\sin \alpha=1$

\section{Das mathematische Problem}

Die mechanische Betrachtung führte auf das rein mathematische Problem, Gleichung (1) zu lösen. Dazu präsentieren wir nun verschiedene, vor allem numerische Methoden. Die Rechnungen wurden auf einem »TI-30 Galaxy«-Taschenrechner durchgeführt; er zeigt 8 Dezimalstellen an. 


\subsection{Analytische Lösung}

Bevor wir uns den numerischen Lösungsmethoden zuwenden, stellen wir kurz die exakte Lösung vor. Interessant dabei ist, dass die hier formal auftretende Hilfsgröße $\varepsilon$ bei einer späteren numerischen Lösung eine geometrische Bedeutung erhält.

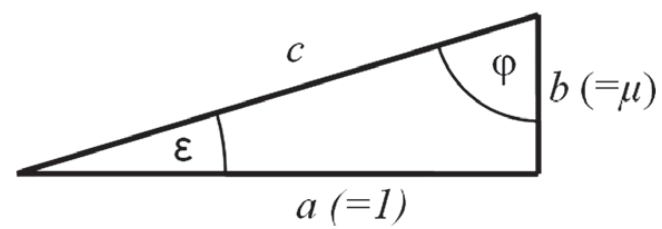

Abb. 5: Interpretation der Koeffizienten der Linearkombination als Dreiecksseiten

In der Linearkombination $a \sin \alpha+b \cos \alpha$ interpretiert man die Koeffizienten a und $b$ als Katheten eines rechtwinkligen Dreiecks (s. Abb. 5). Erweitert man die Linearkombination mit der Hypotenuse $c=\sqrt{a^{2}+b^{2}}$, also $c\left(\frac{a}{c} \sin \alpha+\frac{b}{c} \cos \alpha\right)$ dann sind die neuen Koeffizienten der Kosinus bzw. Sinus des Winkels $\varepsilon$ in dem Dreieck. Das Additionstheorem des Sinus liefert $c(\cos \varepsilon \sin \alpha+\sin \varepsilon \cos \alpha)=c \sin (\alpha+\varepsilon)$. Damit erhält Gleichung (1) die Gestalt

$1=c \sin (\alpha+\varepsilon)$ mit $c=\sqrt{1^{2}+\mu^{2}}$ und $\varepsilon=\arctan \mu$

Für den konkreten Reibungskoeffizienten $\boldsymbol{\mu}=0,1$ er-

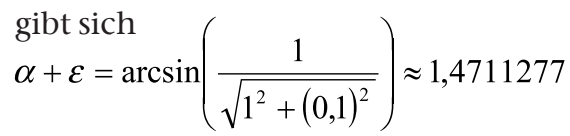

Dem Dreieck in Abb. 5 entnehmen wir $\varepsilon=\arctan 0,1$ $\approx 0,0996687$ und erhalten:

$\alpha \approx 1,4711277-0,0996687=1,3714590\left(\approx 78,58^{\circ}\right)$.

Anmerkung: Statt des Sinus-Additionstheorems kann man auch das Kosinus-Additionstheorem verwenden und erhält

$\cos (\varphi-\alpha)=\frac{1}{\sqrt{1+\mu^{2}}}$

\subsection{Zeichnerische Lösung}

As already stated, sketching functions realistically is crucial for understanding most nonlinear numerical problems.

Acton (2005, S. 4)

Für die erste Übersichtsgraphik verwenden wir die zu Gleichung (1) äquivalente Gleichung

$1-\sin \alpha=0,1 \cos \alpha \quad$ mit $0<\alpha<\pi / 2$

Sie bietet den Vorteil, dass beide Gleichungsseiten wohlbekannte und leicht zu zeichnende Funktionen sind (s. Abb. 6). Aufgrund des Krümmungsverhaltens gibt es zwei Schnittpunkte - den Extremfall bei $\alpha=\pi / 2$ und den gesuchten Grenzwinkel zwischen 1,2 und 1,4.
Abb. 6 zeigt, dass die beiden Kurven im Schnittpunktbereich nahezu parallel verlaufen - daher kann der Schnittpunkt zunächst nur ungenau abgelesen werden. Die geometrische Parallelität ist ein Hinweis auf Schwierigkeiten bei numerischen Verfahren.

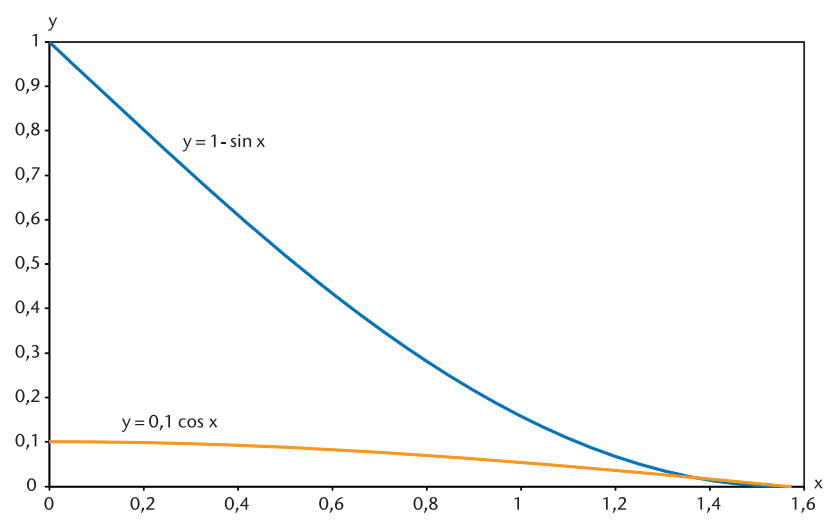

Abb. 6: Übersichtsdarstellung der Gleichung als Schnittpunktproblem

Die Vergrößerung des relevanten Ausschnitts in Abb. 7 liefert bereits eine Unter- und eine Obergrenze für den gesuchten Schnittpunkt mit praktisch brauchbarer Genauigkeit:

$\alpha_{\mathrm{u}}=180^{\circ} / \pi \cdot 1,36=77,9^{\circ}$ und $\alpha_{\mathrm{o}}=180^{\circ} / \pi \cdot 1,38=79,1^{\circ}$.

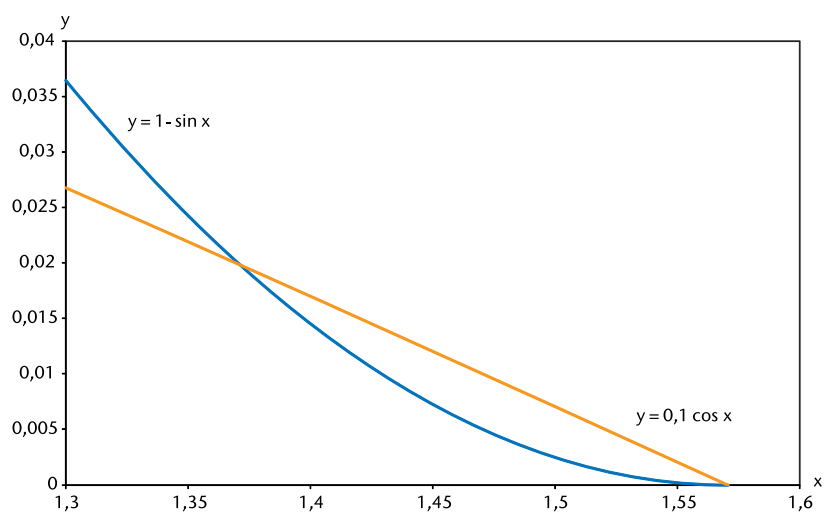

Abb. 7: Vergrößerung des Schnittpunktbereichs

\subsection{Bisektionsverfahren}

Da am gesuchten Schnittpunkt die Größenrelation zwischen den beiden Funktionen wechselt, kann man mit dem einfachen Bisektionsverfahren den Schnittpunkt einschachteln.

Wir starten mit $\alpha_{0}=1,36$ und $\alpha_{1}=1,37$, so dass einmal die subtrahierte Sinuskurve und einmal die skalierte Kosinuskurve den größeren Funktionswert liefert. Der Schnittpunkt liegt im Intervall $\left(\alpha_{0}, \alpha_{1}\right)$. Wir bestimmen die Funktionswerte am Mittelpunkt des Intervalls und entscheiden anhand der Größenbeziehung, mit welchem Teilintervall das Verfahren fortgesetzt wird. So erhält man die folgende Tabelle: 


\begin{tabular}{|l|l|l|l|l|l|}
\hline$n$ & $\alpha_{n}$ & $1-\sin \alpha_{n}$ & $0,1 \cos \alpha_{n}$ & $\begin{array}{c}\text { Rela- } \\
\text { tion }\end{array}$ & $\begin{array}{l}\text { Schnitt- } \\
\text { stelle in }\end{array}$ \\
\hline 0 & 1,37 & 0,0200919 & 0,0199450 & $>$ & \\
\hline 1 & 1,38 & 0,0181465 & 0,0189641 & $<$ & {$\left[\alpha_{0}, \alpha_{1}\right]$} \\
\hline 2 & 1,375 & 0,0191069 & 0,0194548 & $<$ & {$\left[\alpha_{0}, \alpha_{2}\right]$} \\
\hline 3 & 1,3725 & 0,0195964 & 0,0196999 & $<$ & {$\left[\alpha_{0}, \alpha_{3}\right]$} \\
\hline 4 & 1,37125 & 0,0198434 & 0,0198225 & $>$ & {$\left[\alpha_{4}, \alpha_{3}\right]$} \\
\hline 5 & 1,371875 & 0,0197197 & 0,0197612 & $<$ & {$\left[\alpha_{4}, \alpha_{5}\right]$} \\
\hline 6 & 1,3715625 & 0,0197815 & 0,0197918 & $<$ & {$\left[\alpha_{4}, \alpha_{6}\right]$} \\
\hline 7 & 1,3714063 & 0,0198124 & 0,0198072 & $>$ & {$\left[\alpha_{7}, \alpha_{6}\right]$} \\
\hline 8 & 1,3714844 & 0,0197970 & 0,0197995 & $<$ & {$\left[\alpha_{7}, \alpha_{8}\right]$} \\
\hline 9 & 1,3714453 & 0,0198047 & 0,0198033 & $>$ & {$\left[\alpha_{9}, \alpha_{8}\right]$} \\
\hline 10 & 1,3714649 & 0,0198008 & 0,0198014 & $>$ & {$\left[\alpha_{9}, \alpha_{10}\right]$} \\
\hline 11 & 1,3714551 & 0,0198028 & 0,0198024 & $>$ & {$\left[\alpha_{11}, \alpha_{10}\right]$} \\
\hline 12 & 1,3714600 & 0,0198018 & 0,0198019 & $<$ & {$\left[\alpha_{11}, \alpha_{12}\right]$} \\
\hline 13 & 1,3714576 & 0,0198023 & 0,0198021 & $>$ & {$\left[\alpha_{13}, \alpha_{12}\right]$} \\
\hline 14 & 1,3714588 & 0,0198020 & 0,0198020 & $=$ & $=\alpha_{14} ?$ \\
\hline & & & &
\end{tabular}

Üblicherweise weist man dem Bisektionsverfahren die folgenden Eigenschaften zu: Das Verfahren konvergiert gegen den gesuchten Wert (sobald man ein geeignetes Startintervall gefunden hat), aber es konvergiert langsam. Man benötigt jeweils 10 Schritte, um drei zusätzliche Nachkommastellen Genauigkeit zu gewinnen.

Die Berechnung zum Wert $\alpha_{14}$ deutet an, dass auch beim Bisektionsverfahren numerische Schwierigkeiten auftreten können. Haben wir dort zufällig die Schnittstelle gefunden? Die Problematik liegt in der endlichen Zahlendarstellung und der Flachheit der zu berechnenden Funktionen. Tatsächlich zeigt der Taschenrechner für alle fünf Winkel von 1,371 4587 bis 1,371 4591 und beide Funktionen denselben Funktionswert 0,019 8020 an, so dass alle diese Winkel im Rahmen der Taschenrechneranzeige als Schnittstellen in Frage kommen. Die Genauigkeit der Lösung des Bisektionsverfahrens wird durch die Geometrie des Problems - es ist »schlecht konditioniert« (Acton, 2005, S. 18ff) -, stärker eingeschränkt als durch die Genauigkeitsbeschränkungen des Taschenrechners.

\subsection{Treppenstufen-Verfahren - Fixpunktiteration All transcendental equations must be solved iteratively.}

Acton (1990, S. 41)

Das »Treppenstufen-Verfahren «, das in Abb. 8 visualisiert wird, bietet ebenfalls eine robuste Annäherung an den Schnittpunkt. Wir beginnen mit einem Näherungswert $\alpha_{0}$ und berechnen den Funktionswert $y_{0}=0,1$ $\cos \alpha_{0}$. Vom Punkt auf der Kosinuskurve $\left(\alpha_{0}, y_{0}\right)$ bewegen wir uns horizontal bis zur Sinuskurve $\left(\alpha_{1}, y_{0}\right)$, wobei $\alpha_{1}$ $=\arcsin \left(1-y_{0}\right)$. Nun bewegen wir uns vertikal bis zum Punkt $\left(\alpha_{1} ; 0,1 \cos \alpha_{1}\right)$ auf der Kosinuskurve. Abb. 8 zeigt wie die wiederholte Anwendung dieses Treppenstufenschritts den gesuchten Wert $\alpha$ mit zunehmender Genauigkeit liefert. Darüber hinaus sieht man, dass das Verfahren für jeden Startwert $\alpha_{0}$ aus dem Intervall $(0, \pi / 2)$ konvergiert. Das ist in Anbetracht des uninteressanten Schnittpunkts bei $\pi / 2$ eine bedeutende Eigenschaft.

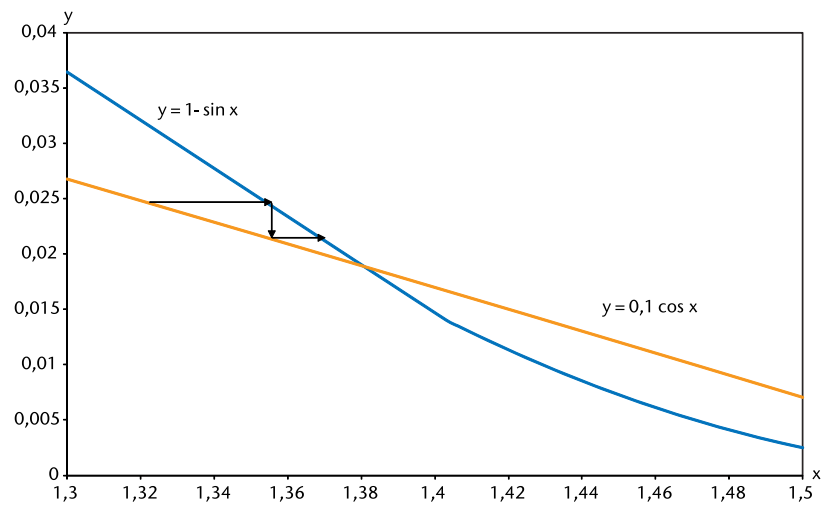

Abb. 8: Visualisierung des Treppenstufen-Verfahrens

Der Startwert $\alpha_{0}=1,37$ liefert beispielsweise

\begin{tabular}{|c|c|c|c|c|c|}
\hline $\mathrm{n}$ & $\alpha_{n}$ & $n$ & $\alpha_{n}$ & $n$ & $\alpha_{n}$ \\
\hline 1 & 1,3707382 & 6 & 1,3714376 & 11 & 1,3714584 \\
\hline 2 & 1,371 1026 & 7 & 1,3714484 & 12 & 1,3714587 \\
\hline 3 & 1,3712827 & 8 & 1,3714538 & 13 & 1,3714589 \\
\hline 4 & 1,371 3717 & 9 & 1,3714564 & 14 & 1,3714589 \\
\hline 5 & 1,3714158 & 10 & 1,3714577 & & \\
\hline
\end{tabular}

Die Iteration konvergiert ebenso langsam wie das Bisektionsverfahren. Die langsame Konvergenz ist eine Folge davon, dass die beiden Kurven in Schnittpunktnähe nahezu parallel verlaufen.

Kombiniert man die beiden Teilformeln des Treppenstufenschritts, so erhält man natürlich die klassische Fixpunktiteration

$\alpha_{\mathrm{n}+1}=\Phi\left(\alpha_{\mathrm{n}}\right) \operatorname{mit} \Phi(\mathrm{x})=\arcsin (1-0,1 \cos \mathrm{x})$

deren Konvergenz aber nicht so offensichtlich ist wie bei der graphischen Darstellung durch Treppenstufen: Die Ableitung

$\Phi^{\prime}(x)=\frac{0,1 \sin x}{\sqrt{1-(1-0,1 \cos x)^{2}}}$

wächst auf dem Intervall $(0, \pi / 2)$ von 0 auf $\infty$. Dass bei dem gesuchten Schnittpunkt die Ableitung kleiner als 1 ist, also die Fixpunktiteration konvergiert, wird erst auf den zweiten Blick deutlich (vgl. Abb. 9). 


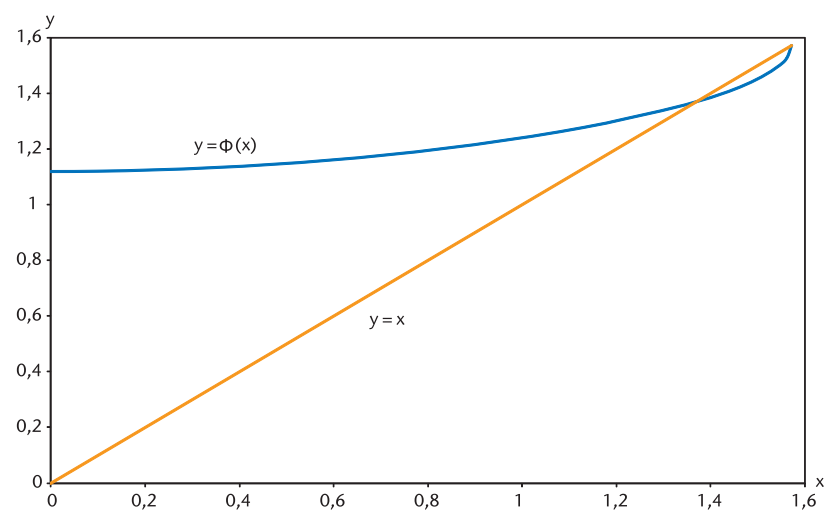

Abb. 9: Funktion der Fixpunktiteration

Die Fixpunktiteration kann die schlechte Konditionierung des Problems nicht beheben. Allerdings kommen für die Fixpunktiteration nur noch drei Winkel im Rahmen der Taschenrechneranzeige als Lösungen in Frage: 1,371 4589; 1,371 4590; 1,371 4591

\subsection{Iteration unter Berücksichtigung der Parallelität} Wir entwickeln nun ein Iterationsverfahren, das die Parallelität der beiden Kurven berücksichtigt. Zunächst berechnen wir dazu die Stelle $\varphi$, an dem sie tatsächlich parallel sind. Gleichsetzen der Ableitungen der beiden Funktionen liefert

$-\cos \varphi=-\mu \sin \varphi$

also für $\boldsymbol{\mu}=0,1$ die Gleichung $\tan \varphi=10$ und den Wert $\varphi \approx 1,4711277$. Dieser Winkel ist der andere spitze Winkel in dem schon betrachteten rechtwinkligen Dreieck in Abb. 5.

Numerisch ist es günstig, kleine Größen zu berechnen. Also setzen wir $\alpha=\varphi-\varepsilon$ in die Gleichung ein und erhalten $1-\sin (\varphi-\varepsilon)=0,1 \cos (\varphi-\varepsilon)$. Die Subtraktionstheoreme liefern

$1-(\sin \varphi \cos \varepsilon-\cos \varphi \sin \varepsilon)=0,1(\cos \varphi \cos \varepsilon+\sin \varphi \sin \varepsilon)$ Umgruppieren nach Funktionen der unbekannten Größe $\varepsilon$ liefert

$1-(\sin \varphi+\mu \cos \varphi) \cos \varepsilon+(\cos \varphi-\mu \sin \varphi) \sin \varepsilon=0$

wobei der zweite Klammerausdruck aufgrund der Definition von $\varphi$ null ist, vgl. Gleichung (2). Wir erhalten

$\cos \varepsilon=\frac{1}{\sin \varphi+\mu \cos \varphi}$

Die trigonometrischen Werte $\sin \varphi=1 / \sqrt{1+\mu^{2}}$ sowie $\cos \varphi=\mu / \sqrt{1+\mu^{2}}$ werden aus dem rechtwinkligen Dreieck in Abb. 5 abgelesen. Einsetzen und Umformen ergibt

$\cos \varepsilon=\frac{1}{\sqrt{1+\mu^{2}}}$
Aufgrund der einfachen Gestalt der Gleichung könnte man nun wie bei der analytischen Lösung in den Taschenrechner einsetzen. Wir wollen stattdessen einen numerischen Zugang verwenden, der auch bei komplizierteren Gleichungsformen zum Erfolg führt. Außerdem hoffen wir, so die schlechte Konditionierung des Problems zu reduzieren.

Für Haftreibungskoeffizienten $\boldsymbol{\mu}$ nahe bei 0 wird die rechte Seite nahe bei 1 liegen. Numerisch ist es günstiger, die kleine Abweichung von 1 als Ausgangsgröße der Berechnung zu verwenden. Damit wird $\varepsilon$ ebenfalls klein sein. Wie nutzen die Taylor-Entwicklungen

$\cos \varepsilon=1-\frac{\varepsilon^{2}}{2 !}+\frac{\varepsilon^{4}}{4 !}-\ldots$

sowie $\frac{1}{\sqrt{1+\mu^{2}}}=1-\frac{1}{2} \mu^{2}+\frac{3}{8} \mu^{4}-\frac{5}{16} \mu^{6}+\ldots$

Da beide Entwicklungen mit 1 starten, kann man nun die jeweiligen Abweichungen miteinander vergleichen. Im Rahmen der Taschenrechnergenauigkeit sind die folgenden Summanden hinreichend.

$\frac{\varepsilon^{2}}{2 !}-\frac{\varepsilon^{4}}{4 !}=\frac{1}{2} \mu^{2}-\frac{3}{8} \mu^{4}+\frac{5}{16} \mu^{6}$

Man vereinfacht über

$\varepsilon^{2}\left(\frac{1}{2}-\frac{\varepsilon^{2}}{24}\right)=\frac{1}{2} \mu^{2}-\frac{3}{8} \mu^{4}+\frac{5}{16} \mu^{6}$

$\mathrm{zu}$

$\varepsilon^{2}\left(12-\varepsilon^{2}\right)=12 \mu^{2}-9 \mu^{4}+7,5 \mu^{6}$

Für $\mu=0,1$ ergibt sich so die Fixpunktiteration für die quadrierte Größe

$\varepsilon_{n+1}^{2}=\frac{0,1191075}{12-\varepsilon_{n}^{2}}$

Die Iteration liefert mit dem natürlichen Startwert $\varepsilon_{0}{ }^{2}=0$ die Werte

\begin{tabular}{|l|l|}
\hline$n$ & $\varepsilon_{n}^{2}$ \\
\hline 1 & 0,0099256 \\
\hline 2 & 0,0099338 \\
\hline 3 & 0,0099338 \\
\hline
\end{tabular}

Letztendlich erhält man $\varepsilon=\sqrt{\varepsilon^{2}}=0,0996687$ und damit auf dem dritten Wege die gesuchte Lösung: $\alpha=\varphi-\varepsilon \approx 1,4711277-0,0996687=1,3714590$

Bei der Iteration liefert der Taschenrechner ein eindeutiges Ergebnis. Die schlechte Konditionierung des Problems steckt in der Wurzeloperation.

Wo liegen die Vorteile des fortgeschrittenen Iterationsansatzes? Man beachte, dass die einfache Iteration erst nach 13 Schritten eine stabile Lösung erreichte und in jedem Schritt eine Kosinus- und eine Arkussinusbe- 
rechnung erforderlich waren. Dahingegen benötigt die fortgeschrittene Variante nur 2 Schritte, die darüber hinaus nur die Grundrechenarten verwenden. Wäre ein ähnliches Problem im Rahmen einer Programmschleife sehr oft wiederholt zu berechnen, so wäre der Zeitaufwand der fortgeschrittenen Methode deutlich geringer.

Schließlich wird bei der Herleitung der fortgeschrittenen Iteration auch der Winkel $\varphi=\alpha+\varepsilon$ mit einer Bedeutung belegt. Er kam als Hilfsgröße schon in der exakten Rechnung vor, schien dort aber eher künstlich und ohne tiefere Bedeutung zu sein.

\subsection{Newton-Verfahren}

Abschließend wenden wir das Newton-Verfahren an. Die Iterationsvorschrift

$x_{n+1}=x_{n}-\frac{1-\sin x_{n}-0,1 \cos x_{n}}{-\cos x_{n}+0,1 \sin x_{n}} \quad$ für $n=1,2,3, \ldots$

benötigt mit dem zeichnerisch gefundenen Startwert $x_{0}$ = 1,37 lediglich drei Iterationen:

\begin{tabular}{|c|c|c|c|c|}
\hline n & $x_{n}$ & $f\left(x_{n}\right)$ & $f^{\prime}\left(x_{n}\right)$ & $f\left(x_{n}\right) / f^{\prime}\left(x_{n}\right)$ \\
\hline 0 & 1,37 & 0,000147 & $-0,1014589$ & $-0,001445$ \\
\hline 1 & 1,3714485 & 0,000001 & $-0,1000105$ & $-0,0000105$ \\
\hline 2 & 1,3714590 & $3,58 \cdot 10^{-10}$ & $-0,1000000$ & $-3,58 \cdot 10^{-9}$ \\
\hline 3 & 1,3714590 & & & \\
\hline
\end{tabular}

Allerdings müssen pro Iteration zwei trigonometrische Funktionswerte berechnet werden. Der fortgeschrittene Iterationsansatz erweist sich weiterhin als effizienter.

Der Erfolg des Newton-Verfahrens hängt darüber hinaus sowohl von einem guten Startwert als auch von einer geeigneten Geometrie im Bereich der Nullstelle ab. Im vorliegenden Problem ist diese Geometrie durchaus kritisch zu sehen. Problematisch ist, dass die Funktion, deren Nullstelle wir suchen, vgl. Abb. 10, in der Nähe der Nullstelle sehr flach verläuft.

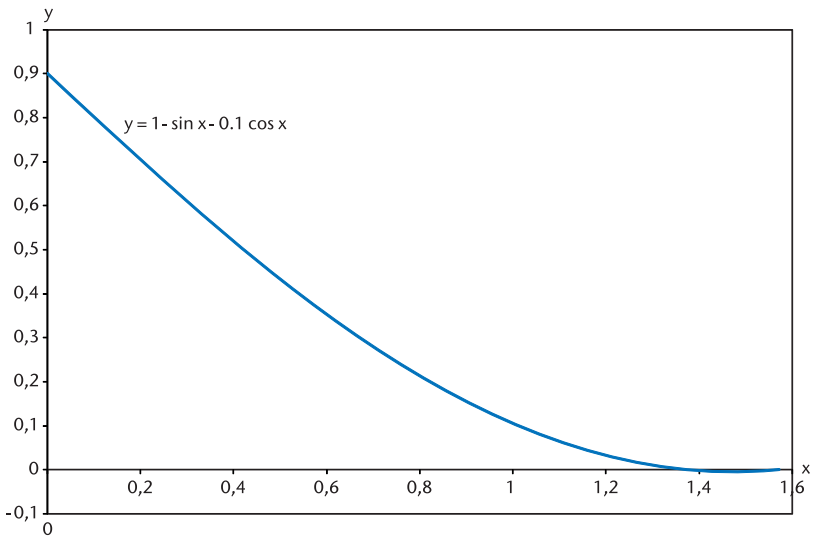

Abb. 10: Darstellung als Nullstellenproblem für Newton-Verfahren
Wählt man einen Startwert aus diesem Bereich, z. B. $\mathrm{x}_{0}=1,47$ so liefert ein Newton-Schritt

$x_{n+1}=1,47-\frac{-0,0049869}{-0,0011333}=1,47-4,4003353=-2,9303353$

womit das Verfahren den Einzugsbereich der gesuchten Nullstelle verlässt. Das Newton-Verfahren liefert nicht für jeden Startwert aus $(0, \pi / 2)$ die gesuchte Lösung. Abb. 10 zeigt, dass für einen Startwert kleiner als die gesuchte Nullstelle das Newton-Verfahren aufgrund der Konvexität der Funktion jedoch zuverlässig konvergieren wird. Erneut zeigt sich die Bedeutung von Zeichnungen im Zusammenhang mit numerischen Verfahren.

\section{Zusammenfassung}

Indeed, a sketch is the best source of good starting values [...] Acton (1990, S. 48)

Zeichnungen sind unerlässlich für numerische Verfahren. Sie bieten einen ersten Überblick über die Lösungen und die zu erwartenden Probleme bei ihrer Berechnung. Sie helfen auch bei der Wahl eines geeigneten Verfahrens. Noch konkreter entnimmt man ihnen die Startwerte, die von vielen Verfahren benötigt werden. Schließlich kann man häufig ersehen, welche Konvergenzbereiche die unterschiedlichen Verfahren besitzen.

Einige weniger bekannte Aspekte numerischer Verfahren wurden am Beispiel vorgestellt: Die Lösung des Bisektionsverfahrens muss in der Praxis nicht eindeutig sein. Ist ein Problem schlecht konditioniert, kann auch die robuste Bisektion daran nichts ändern. Die Fixpunktiteration kann als Treppenstufenverfahren einfach visualisiert werden. Dabei kann die Iteration wesentlich optimiert werden, wenn man das Problem mit Hilfe kleiner Größen formuliert. Die Treppenstufeniteration und ihre Optimierung zeichnen sich gegenüber dem bekannteren Newton-Verfahren dadurch aus, dass die Wahl des Startwertes weniger problematisch ist. Die Konvergenz ist im Beispiel sogar global und für das optimierte Verfahren existiert der natürliche Startwert 0. Abschließend sei noch erwähnt, dass der Rechenaufwand beim optimierten Treppenstufenverfahren minimal ist - es werden nur Grundrechenarten statt aufwändiger Funktionsauswertungen benötigt. Bei Berechnungen innerhalb häufig zu durchlaufender Schleifen ist das eine wichtige Eigenschaft. 


\section{Literatur}

Acton, F. (2005). Real Computing Made Real - Preventing Errors in Scientific and Engineering Calculations. Dover Publications, Mineola, New York

Acton, F. (1990). Numerical Methods that work. Mathematical Association of America

Hibbeler, R. C. (2004). Statics \& Dynamics. Pearson Prentice Hall, Upper Saddle River, $10^{\text {th }} \mathrm{e}$

\section{Autoren}

Rainer Berghaus

Fachbereich Ingenieurwesen/Wirtschaftsingenieurwesen

Technische Hochschule Wildau [FH]

Fichtestraße 97, 15745 Wildau

Dr. (USA) Achim Kehrein

Fachbereich Ingenieurwesen/Wirtschaftsingenieurwesen

Technische Hochschule Wildau [FH]

achim.kehrein@th-wildau.de 